\title{
The effect of delayed adjuvant chemotherapy on relapse of triple- negative breast cancer
}

\author{
Shuang $\mathrm{Li}^{1 \#}$, Ding Ma ${ }^{2 \#}$, Hao-Hong Shi ${ }^{3 \#}$, Ke-Da Yu ${ }^{2}$, Qiang Zhang ${ }^{1}$ \\ ${ }^{1}$ Department of Breast Surgery, Liaoning Cancer Hospital and Institute, Shenyang 110042, China; ${ }^{2}$ Department of Breast Surgery, Shanghai Cancer \\ Center and Cancer Institute, ${ }^{3}$ Department of Anesthesiology, Children's Hospital, Shanghai Medical College, Fudan University, Shanghai 200032, \\ China \\ Contributions: (I) Conception and design: Q Zhang, KD Yu; (II) Administrative support: Q Zhang, KD Yu; (III) Provision of study materials or \\ patients: S Li, KD Yu; (IV) Collection and assembly of data: S Li, HH Shi; (V) Data analysis and interpretation: S Li, D Ma, KD Yu; (VI) Manuscript \\ writing: All authors; (VII) Final approval of manuscript: All authors. \\ "These authors contributed equally to this work. \\ Correspondence to: Ke-Da Yu, MD, PhD. Department of Breast Surgery, Shanghai Cancer Center and Cancer Institute, Shanghai Medical College, \\ Fudan University, Shanghai 200032, China. Email: yukeda@fudan.edu.cn; Qiang Zhang, MD. Department of Breast Surgery, Liaoning Cancer \\ Hospital and Institute, Shenyang 110042, China. Email: zhangqiaoln@163.com.
}

\begin{abstract}
Background: For triple negative breast cancer (TNBC), the optimal time from surgery to initiation of adjuvant chemotherapy is controversial. We investigated the influence of time to adjuvant chemotherapy on outcome in TNBC patients.

Methods: Female patients with stage I-IIIa operable TNBC between 2006 and 2008 in our institutions were included. A total of 331 patients were divided into 3 groups according to the time to adjuvant chemotherapy: $\leq 30,31-60$, and $>60$ days. Relapse free survival (RFS) were calculated and compared.

Results: Prolonged delay of initiation of adjuvant chemotherapy ( $\leq 30$ versus $>60$ days) significantly decreased the RFS in our TNBC cohort [adjusted hazard ratio (HR) of 2.39; $95 \%$ confidence interval (CI), $1.13-5.07, \mathrm{P}=0.02]$. While a moderate delay ( $\leq 30$ versus $31-60$ days) did not significantly influence RFS in all TNBC patients, it did compromise survival in lymph node positive patients $(\mathrm{P}=0.04)$.

Conclusions: Longer delay of adjuvant chemotherapy was associated with worse survival in TNBC patients. Early initiation of adjuvant chemotherapy should be considered, especially for relatively high risk node positive TNBCs.
\end{abstract}

Keywords: Timing; adjuvant chemotherapy; triple-negative breast cancer; survival

Submitted Dec 12, 2017. Accepted for publication Apr 04, 2018.

doi: $10.21037 /$ jtd.2018.04.94

View this article at: http://dx.doi.org/10.21037/jtd.2018.04.94

\section{Introduction}

Triple-negative breast cancer (TNBC), defined by lacking estrogen receptor (ER), progesterone receptor (PR) expression as well as human epithelial growth receptor 2 (HER2) gene amplification, consist of a group of biologically aggressive and highly proliferative disease. Chemotherapy has been accepted as the mainstay of systemic treatment for TNBC patients, bringing dramatic benefit to both early and late stage TNBC patients (1). But
TNBC still has a higher rate of loco-regional and distant recurrence and a worse prognosis than women with other breast cancer subtypes. Optimization in both chemotherapy regimen and how we give them is high priority.

Currently, $60 \%$ of all the breast cancer patients and more than $80 \%$ TNBC are routinely given adjuvant chemotherapy, while, however, the optimal time point to start adjuvant chemotherapy remains controversial. Researches have demonstrated that tumor resection and surgical trauma might accelerate the growth of distant 
Table 1 Triple-negative breast cancer patient characteristics by time from surgery to adjuvant chemotherapy

\begin{tabular}{|c|c|c|c|c|c|c|c|c|c|}
\hline \multirow{2}{*}{ Characteristics } & \multicolumn{8}{|c|}{ Interval from surgery to chemotherapy initiation (days) } & \multirow{2}{*}{$\mathrm{P}^{\#}$} \\
\hline & $\mathrm{n}$ & $\%$ & $\mathrm{n}$ & $\%$ & $\mathrm{n}$ & $\%$ & $\mathrm{n}$ & $\%$ & \\
\hline Median age, years & 48 & - & 47 & - & 49 & - & 48 & - & 0.6 \\
\hline Tumor size, $T$ category & & & & & & & & & 0.5 \\
\hline $\mathrm{T} 2$ & 198 & 60 & 128 & 59 & 60 & 63 & 10 & 50 & \\
\hline T3 & 38 & 11 & 22 & 10 & 12 & 13 & 4 & 20 & \\
\hline Lymph node status, $\mathrm{N}$ category & & & & & & & & & 0.6 \\
\hline No & 147 & 44 & 101 & 47 & 36 & 38 & 10 & 50 & \\
\hline
\end{tabular}

Because data for tumor grade were lacking in many cases, we did not include this variable in our analysis. Tumor stage could be judged by tumor size and lymph node status. ", P values for differences in three groups are tested by ANOVA or heterogeneous $\chi^{2}$.

micro-metastasis, supporting the early initiation of adjuvant chemotherapy (2). Despite these biological rationale, several studies retrospectively comparing early and delayed starting of adjuvant chemotherapy have published conflicting outcome (3-7). This might be explained by differences in patient selection, disease characteristics as well as arbitrary choice in delay time truncation.

With regards to the TNBC, limited data have presented the effect of delayed initiation of chemotherapy on survival or relapse. A recent study including approximately 25 thousands breast cancer patients reported that delaying initiation of adjuvant chemotherapy over 90 days can result in dismal outcomes (8). Notably, delayed time to adjuvant chemotherapy was particularly detrimental among TNBCs. Another piece of evidence also showed that prolonged delay of chemotherapy was related to worse prognosis, supporting the early start of adjuvant chemotherapy in patients with aggressive breast cancer subtypes, i.e., TNBC $(9,10)$. To further confirm those amature findings, we perform this retrospective analysis specifically in TNBC to provide additional evidence.

\section{Methods}

\section{Patients}

We retrospectively studied the cases recorded by the Department of Breast Surgery at Liaoning Cancer Hospital and the Department of Breast Surgery at Fudan University Shanghai Cancer Center (FUSCC). Detailed description of this patient cohort can be found in our previous publications $(11,12)$. This study was approved by the Ethical Committees of the FUSCC and Liaoning Cancer Hospital, each participant has signed an informed consent form.

From 2006 to 2008, 465 consecutive female patients diagnosed as stage I to stage IIIa operable TNBC were treated surgically (including breast conservation surgery, mastectomy and modified radical operation) at Liaoning Cancer Hospital and Shanghai Cancer Center. The preoperative assessment (both routine chemical and imaging examination), surgery, pathological evaluation [gross and microscopic examination, immunohistochemistry (IHC), in-situ hybridization], and adjuvant treatment regimen had already been reported in detail elsewhere (12). Seventy-two of these patients were ruled out for the following reasons: 61 not treated with adjuvant chemotherapy, 11 only receiving oral chemotherapeutic regimen like capecitabine. The chemotherapy regimens used in this study were either anthracycline-based or taxane-based. Among the remaining ones, 62 cases were further excluded due to insufficient cycle number ( $\leq 3$ cycles). Finally, 331 TNBC patients constituted our analysis cohort. Overview of the clinical and pathological characteristics of this cohort can be found in Table 1. 


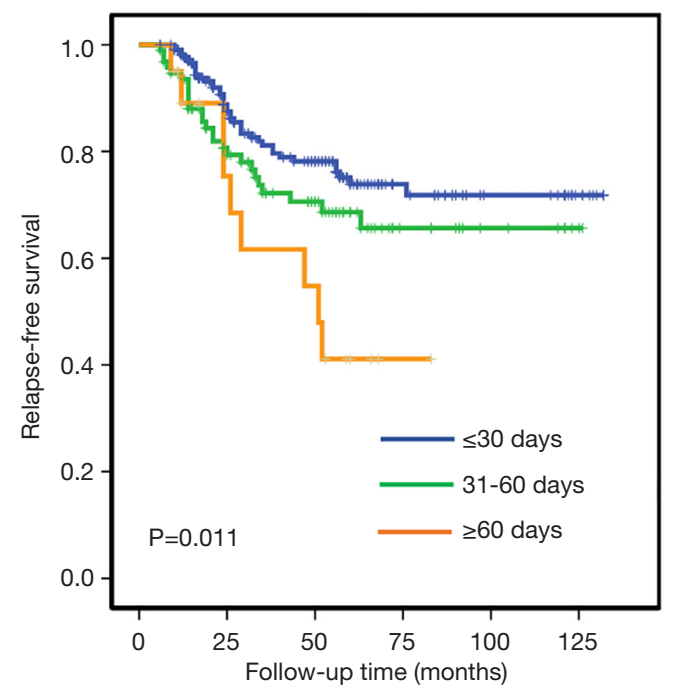

Figure $1 \mathrm{KM}$ plot for RFS according to time between surgery and adjuvant chemotherapy. KM curves for three groups: 1 to 30 days, 31 to 60 days, and longer than 60 days from definitive surgery to start of adjuvant chemotherapy. KM, Kaplan-Meier; RFS, relapse free survival.

\section{Determination of TNBC}

Status of both hormone receptors (ER and PR), and HER2 were evaluation at the pathology centers in the Department of pathology of Liaoning Cancer Hospital and Shanghai Cancer Center with standard procedures. As for ER and/or $\mathrm{PR}$, positivity required equal or more than $10 \%$ positively stained tumor cells as evaluated by IHC. HER2 positive was defined as protein over expression (IHC $2+$ ) and/or $E R B B 2$ gene amplification detected by fluorescence in situ hybridization (FISH). TNBC was defined as ER-negative, PR-negative, and HER2-negative.

\section{Survival analysis and statistics}

We grouped the patients by the time from breast cancer surgery to adjuvant chemotherapy: less than 31 days as group 1 (216 cases), 31-60 days as group 2 (95 cases), and over 60 days as group 3 (20 cases). The main results of interest are relapse-free survivals (RFS), defined as the time from surgery to either local/regional recurrence, metastasis or in situ/contralateral breast cancer. To ensure the reliability of relapse status, all patients were followed up for $\geq 6$ months and the median follow-up was 91 months. Kaplan-Meier (KM) method and log-rank test were performed to build and compare survival curves, respectively.
Cox proportional hazards regression models were also adopted to perform multivariate survival analyses with adjustment for confounders. Statistics mentioned above were conducted using Statistical Package for the Social Sciences (SPSS) version 20 (SPSS Incorporated, Chicago, IL, USA) or Stata version 14.0 (StataCorp, College Station, TX, USA), where bilateral $\mathrm{P}<0.05$ was considered as statistically significant.

\section{Results}

We studied the association between adjuvant chemotherapy initiation time and RFS in 331 patients with stage I-IIIa operable TNBC. While about two-thirds $(65.3 \%)$ of the studied patients started chemotherapy within 30 days, a few patients $(6.0 \%)$ received no adjuvant chemotherapy until 61 or even more days after surgery. Clinical and pathological characteristics collected before the first cycle of chemotherapy distributed equally between the studied groups, as demonstrated in Table 1 . The 5 -year RFS rates were $74 \%, 65 \%$ and $40 \%$ in group 1 (start chemotherapy in no more than 30 days since surgery), group 2 (start chemotherapy in 31-60 days), and group 3 (no chemotherapy until 61 or more days), respectively ( $\mathrm{P}<0.001$ for the heterogeneity among the 3 groups).

In univariate analysis, both group 1 and group 2 were found to have significantly better RFS comparing with group 3, while no significant but only numerical difference were seen in RFS between group 1 and group 2 (comparison between group 1 and group 2, log-rank $\mathrm{P}=0.12$; between group 1 and group 3, log-rank $\mathrm{P}=0.004$; Figure 1).

We further conducted multivariate analysis (demonstrated in Table 2), and found that both lymph node status and time to adjuvant chemotherapy were independent prognostic factors for RFS. Prolonged delay of chemotherapy over 60 days significantly reduced RFS (HR 2.39; 95\% CI, 1.13-5.07, $\mathrm{P}=0.02$ ) compared with early initiation of adjuvant chemotherapy after adjustment of other prognostic factors. However, a modest delay (31-60 days delay versus $\leq 30$ days delay) would not compromise RFS in the whole TNBC population (HR 1.42; 95\% CI, 0.86-2.33, $\mathrm{P}=0.17$ ).

To further investigate whether a modest delay (31-60 days delay versus $\leq 30$ days delay) would compromise survival in the high-risk TNBC, we made a stratification analysis according to the lymph status. The stratification analysis indicated that a modest delay might reduce the RFS in node-positive TNBC patients $(\mathrm{P}=0.04)$, rather than in those 
Table 2 Prognostic factors for RFS of TNBC in multivariate analysis

\begin{tabular}{lccc}
\hline \multirow{2}{*}{ Factor } & \multicolumn{2}{c}{$\mathrm{RFS}$} & \multirow{2}{*}{$\mathrm{P}$} \\
\cline { 2 - 3 } & $\mathrm{HR} \mathrm{H}^{*}$ & $95.0 \% \mathrm{Cl}$ & \\
\hline Age & 1.00 & $0.98-1.03$ & 0.79 \\
$\begin{array}{l}\text { Size } \\
\text { T0-T1 vs. T2 }\end{array}$ & 1.12 & $0.62-2.05$ & 0.69 \\
$\quad$ T0-T1 vs. T3-4 & 1.31 & $0.63-2.73$ & 0.47 \\
Nodal status & & & \\
$\quad$ N0 vs. N1 & 2.53 & $1.16-5.55$ & 0.02 \\
$\quad$ N0 vs. N2 & 7.94 & $4.06-15.53$ & $<0.001$ \\
Time to chemotherapy (days) & & & \\
$\quad \leq 30$ vs. 31-60 & 1.42 & $0.86-2.33$ & 0.17 \\
$\quad \leq 30$ vs. >60 & 2.39 & $1.13-5.07$ & 0.02 \\
\hline
\end{tabular}

Method for Cox proportional hazards analysis: Enter all the variables. *, Adjusted for all the factors listed in the table. $\mathrm{Cl}$, confidence interval; HR, hazard ratio; TNBC, triple-negative breast cancer; RFS, relapse free survival.

node-negative women $(\mathrm{P}=0.27)$. A stratification analysis according to tumor size also demonstrated that modest delay of adjuvant chemotherapy tended to compromise RFS in patients with tumor size $>2 \mathrm{~cm}(\mathrm{P}=0.06)$, but not in T1 patients $(\mathrm{P}=0.21)$. These results indicated the necessity of early initiation of adjuvant chemotherapy in high-risk TNBC.

\section{Discussion}

The exact timing for adjuvant chemotherapy after breast cancer surgery to achieve maximum survival benefit is controversial. Previous published clinical trials did not clearly recommend the timing of postoperative chemotherapy. In this study, we add to the evidence that the duration between surgery and adjuvant chemotherapy would influence the prognosis of TNBC, an aggressive breast cancer subtype characterized by early recurrence and lack of effective systemic treatment other than chemotherapy. Our study shows that delayed chemotherapy could result in worse outcome of TNBC patients, especially node positive ones.

The previous analysis of the trials of the international breast cancer team demonstrated that early initiation of adjuvant chemotherapy can improve the survival of premenopausal ER- breast cancer patients rather than those with ER+ tumors (3). Two recent studies also linked late adjuvant chemotherapy initiation to poor prognosis in both HER2 + patients (treated with or without trastuzumab) and TNBC patients, in support of the notion that delayed adjuvant chemotherapy may be especially harmful for patients with aggressive breast tumors $(8,9)$. Moreover, Chavez-MacGregor and his colleagues also found that longer time to chemotherapy caused TNBC patients to have worse overall survival (OS) as well as worse breast cancer-specific survival (BCSS) (8). Our results, in line with these studies, suggested that patients with TNBC should begin adjuvant chemotherapy soon after surgery. Moreover, to further investigate whether a modest delay (31-60 days delay versus $\leq 30$ days delay) would compromise survival in the high-risk TNBC, we made a stratification analysis according the lymph status. The results indicated that node-positive TNBC, rather than relatively low risk nodenegative disease, may benefit from a very early initiation ( $\leq 30$ days) of chemotherapy.

Our research has several limitations. First, this study was retrospective, nonrandomized, and limited in information on clinical characteristics. Our results need to be further validated in independent cohort. In fact, chemotherapy dose, cycle numbers and completion rates are key determinants of patient survival and should be taken into consideration in this kind of studies. However, the retrospective nature of the data made the information insufficient. Second, because of the low frequent of TNBC in the whole population, 331 cases are still small sample sizes for powerful statistical analysis, and only $6 \%$ patients (20 in 331) had delay over 60 days. In addition, longer follow-up time is necessary and OS should be further evaluated.

In conclusion, our findings gave evidence for the association between prolonged adjuvant chemotherapy initiation and poorer prognosis in TNBC. Although initiation of adjuvant chemotherapy within 60 days is the adequate time windows for most TNBC cases, an earlier initiation within 30 days might be more helpful for those TNBC patients with extremely high-risk factors.

\section{Acknowledgements}

Funding: This work was supported by grants from the National Natural Science Foundation of China [81572583, 81672600, 81722032].

\section{Footnote}

Conflicts of Interest: The funders of this study had no role in the study design, data collection and analysis, decision to publish, or preparation of the manuscript. 
Ethical Statement: This study was approved by the Ethical Committees of the FUSCC and Liaoning Cancer Hospital (approval ID: 1603158-Exp4), each participant has signed an informed consent form.

\section{References}

1. Foulkes WD, Smith IE, Reis-Filho JS. Triple-negative breast cancer. N Engl J Med 2010;363:1938-48.

2. Gunduz N, Fisher B, Saffer EA. Effect of surgical removal on the growth and kinetics of residual tumor. Cancer Res 1979;39:3861-5.

3. Colleoni M, Bonetti M, Coates AS, et al. Early start of adjuvant chemotherapy may improve treatment outcome for premenopausal breast cancer patients with tumors not expressing estrogen receptors. The International Breast Cancer Study Group. J Clin Oncol 2000;18:584-90.

4. Shannon C, Ashley S, Smith IE. Does timing of adjuvant chemotherapy for early breast cancer influence survival? J Clin Oncol 2003;21:3792-7.

5. Fedewa SA, Ward EM, Stewart AK, et al. Delays in adjuvant chemotherapy treatment among patients with breast cancer are more likely in African American and Hispanic populations: a national cohort study 2004-2006. J Clin Oncol 2010;28:4135-41.

Cite this article as: Li S, Ma D, Shi HH, Yu KD, Zhang Q. The effect of delayed adjuvant chemotherapy on relapse of triple-negative breast cancer. J Thorac Dis 2018;10(5):28372841. doi: $10.21037 /$ jtd.2018.04.94
6. Alkis N, Durnali AG, Arslan UY, et al. Optimal timing of adjuvant treatment in patients with early breast cancer. Med Oncol 2011;28:1255-9.

7. Nurgalieva ZZ, Franzini L, Morgan RO, et al. Impact of timing of adjuvant chemotherapy initiation and completion after surgery on racial disparities in survival among women with breast cancer. Med Oncol 2013;30:419.

8. Chavez-MacGregor M, Clarke CA, Lichtensztajn DY, et al. Delayed Initiation of Adjuvant Chemotherapy Among Patients With Breast Cancer. JAMA Oncol 2016;2:322-9.

9. Yu KD, Fan L, Qiu LX, et al. Influence of delayed initiation of adjuvant chemotherapy on breast cancer survival is subtype-dependent. Oncotarget 2017;8:46549-56.

10. Gagliato Dde M, Gonzalez-Angulo AM, Lei X, et al. Clinical impact of delaying initiation of adjuvant chemotherapy in patients with breast cancer. J Clin Oncol 2014;32:735-44.

11. Yu KD, Li S, Shao ZM. Different annual recurrence pattern between lumpectomy and mastectomy: implication for breast cancer surveillance after breast-conserving surgery. Oncologist 2011;16:1101-10.

12. Yu KD, Di GH, Wu J, et al. Development and trends of surgical modalities for breast cancer in China: a review of 16-year data. Ann Surg Oncol 2007;14:2502-9. 